\title{
Pediatrik Robotik Üroloji
}

\author{
Bülent Önal, Çağatay Doğan
}

İstanbul Üniversitesi, Cerrahpaşa Tıp Fakültesi, Üroloji Anabilim Dalı, İstanbul

\section{Giriş}

5 ocuklara yönelik cerrahi girişimlerin kompleks ve rekonstrüktif doğası ve doğmamış fetus ve infantlardan büyük çocuk ve adolesanlara kadar uzanan hasta spektrumu; yüksek cerrahi görüş, beceri ve doğruluk gerektirir. Derinlik algısı, yüksek büyütmedeki sabit görsel operasyon alanını doğrudan kontrol etme yeteneği, hareket özgürlüğ̈u, hareket sırasında mükemmel göz ve el koordinasyonunun yanı sıra; yüksek ergonomi ile elde edilmiş üç boyutlu panoramik yüksek çözünürlüklü görüntülerin elde edilmesi, pediatrik ve neonatal cerrahi için robotu ideale yakın bir araç haline getirmektedir. Açık cerrahi ile karşılaştırıldığında robotik cerrahide kısa hospitalizasyon süresi, minimal narkotik analjezi gereksinimi ve daha iyi kozmetik sonuçlar ön plana çıkmaktadır. Nguyen ve ark.'ları postoperatif insizyon skarı için yaptıkları çalışmada; üreteral reimplantasyon, piyeloplasti ve mesane augmentasyonunda skar durumunun, aile ve çocuk için önemli olduğunu rapor etmişlerdir (1).

Robotik sistem, minimal invaziv cerrahinin doğasındaki zorlukların birçoğunun üstesinden gelmiştir ve eskiden tekniği zor olarak görülen kompleks cerrahi işlemlerin yap1labilme becerisini artırmıştır (2-4). Ancak, bu sistemin de sınırlamaları yok değildir. Dokunma duyusunun tamamen ortadan kalkması, pediatrik hassas dokularla ve mikrosütür materyalleri ile çalışırken cerrahın fazladan özen göstermesini gerektirir. Diğer dezavantajlar ise; kullanılan enstrümanların boyutundan ortaya çıkmaktadır. Robotun boyutu ve kapladığı alan anestezistin hastaya ulaşmasını kısıtlayabilir (üst abdomen ve toraks işlemlerinde, küçük çocuklarda) ve hastanın içinde ve dışında kol - araç çarpışmalarına neden olabilir (infantlar ve küçük çocuklarda) (5). Diğer taraftan robotik aletlerin minyatürizasyonu konusunda çalışmalar devam etmektedir. Yeni geliştirilen $8 \mathrm{~mm}$ 'lik teleskop, 12 mm'lik teleskopun üzerine çıkan bir gelişmedir. Hem 5 hem de $8 \mathrm{~mm}$ 'lik aletler efektiftir ancak daha küçük aletler şüphesiz cerrahın daha küçük alanlarda çalışma kabiliyetini artırır ve cerrahi travmayı da azaltır. Bazı yazarlar 7 yaşın altındaki çocuklarda $5 \mathrm{~mm}$ 'lik trokarları önermektedirler (6). Küçük çocuklarda özellikle infantlarda pnömoperitonyum için abdomen sınırlı miktarda gaz (yaklaşık 1-2 litre kadar) tutabilir ve Karın içi basınç $10 \mathrm{~mm} \mathrm{Hg}$ seviyesinde tutulmaya çalışılır (7). Hem abdomendeki alan kısıtlığı, hem de çocukların trokarları yerinde tutacak batın katlarının inceliğinden dolayı, portlar alet değişimi sırasında her an çıkabilir. Bu nedenle bazı yazarlar trokarları batın duvarına sabitlemeyi önerir (8). Ancak genel olarak geniş olmayan uygun cilt insiz- yonu trokarı yerinde tutar ve tespite gerek kalmaz.

Preoperatif orogastrik tüp ve idrar sondası intraabdominal alanı artırmak ve visseral organ yaralanmasının önüne geçmek için gereklidir. Transperitoneal yaklaşımda Hasson açık giriş tekniği, pnömoperitonyum sağlamada abdominal organ ve büyük damarları yaralamadan güvenli olarak intraabdominal kaviteye giriş sağlar. Robot portları çarpışmaları önlemek için kamera portuna ve hedef organa eşit uzaklıkta yerleştirilmelidir. Doğru port yerleşimi başarı için kritik öneme sahiptir.

Çocuk ürolojisindeki kompleks rekonstrüktif girişimlere geçmeden başlangıçta yeterli deneyim kazanmak için öncelikle varikosel ligasyonu ve nefrektomi önerilir (7). Artan deneyim ile bir sonraki basamak pyeloplasti ve sonra daha karmaşık rekonstrüktif girişimler olmalıdır. Meininger ve ark.'ları 2001'de ilk kez pediatrik yaş grubunda robot kullanımı ile ilgili 2 olguluk Nissen fundiplikasyon deneyimlerini rapor etmişlerdir (9). Bunu takiben Peters ve ark.'ları 2002'de robot ile ilk ürolojik girişim olan piyeloplasti deneyimlerini rapor etmişlerdir (10). 2001-2012 yılları arasında yayınlanan pediatrik robotik cerrahide genitoüriner, gastrointestinal ve torasik girişimlerde en sik piyeloplasti $(n=672)$, fundiplikasyon $(n=424)$ ve lobektomi $(n=18)$ rapor edilmiştir $(11)$. Bugüne kadar yayınlanan en geniş seri Kasturi ve ark.'larının 150 hastada uyguladıkları 300 ekstravezikal üreteral reimplantasyon girişimidir (12). Genel olarak, robotik cerrahiden açı̆̆a geçiş oranı \%2.5 olarak belirtilmiştir ve bu oran genitoüriner, gastrointestinal ve torasik girişimlerde sırasıyla \%1.3, $\% 3.9$ ve $\% 10$ 'dur.

\section{Piyeloplasti}

Klasik açı Anderson-Hynes piyeloplasti girişimi \%90100 başarı oranıyla altın standarttır (13). Dismembered piyeloplasti yapılan çalışmalarda diğer girişimlere göre daha üstün bulunmuştur $(14,15)$. Üreteropelvik bileşke darlıklarinda laparoskopik piyeloplasti ilk kez 1993'de Schuessler ve ark.'ları tarafından tanımlanmıştır (16). Bunu takiben birçok seride fonksiyonel sonuçlar açık cerrahi ile benzer bulunmuştur (17). Laparoskopik piyeloplasti teknik gereksinimlerden dolayı özellikle intrakorporeal sutur gereksinimi nedeniyle çocuklarda yaygın kullanım alanı bulamamıştır (18). Laparoskopik cerrahide, eğitim ve deneyim bir tarafa, sağlanan iki boyutlu görüntü nedeniyle enstrümanların hareketi ve dokuların manipülasyonu zordur.

Da Vinci ${ }^{\circledR}$ sisteminin kullanılmaya başlanması ile birlikte, robotik pyeloplasti alternatif ve uygun bir seçenek olarak 
ortaya çıkmıştır. Yukarıda belirtildiği şekilde, çocuklarda en sık kullanılan robotik yardımlı laparoskopik girişimdir (19). Üç boyutlu görüntü, yedi derece enstrümanların hareket özgürlüğü ile açık cerrahideki manipülasyonları taklit edebilmesi, hareketlerde tremorun olmaması robotu laparoskopiden daha ergonomik hale getirir. Popülaritesine rağmen uzun dönem sonuçlar içeren seri sayısı azdır. Buna ek olarak çeşitli cerrahi yöntemler arasında karşılaştırma yapan çalışma sayısı sinırlıdır ve çoğu retrospektiftir. Şu anki literatür sonuçlarında açı dismembered, laparoskopik ve robotik yardımlı laparoskopik yöntemlerin uzun dönem sonuçları benzerdir (20). Ancak açık cerrahi ile karşılaştırıldığında laparoskopik ve robot yardımlı laparoskopik yöntemde daha az ve kısa süreli analjezik ihtiyacı ve daha kısa hospitalizasyon süresinin olduğu gösterilmiştir (21-23). Açık ile karş1laştırıldığında robot yardımlı laparoskopik piyeloplastide operasyon süresi daha uzundur. Ancak daha sonra yapılan çalışmalarda cerrah, anestezist ve hemşire arasında işbirliğinin pekişmesi ile operasyon süresi anlamlı olarak azalmış açı cerrahiye yaklaşmışır $(23,24)$. Ayrıca robot yardımlı laparoskopik piyeloplasti ile açık cerrahi karşılaştırıldığında genel yaşam tatmini, cerrahinin hastanın yaşamına olumlu etkisi, postoperatif takip yükünde azalma, insizyon skarı konusunda aile memnuniyeti daha yüksek bulunmuştur (25). Laparoskopi ile robot yardımlı laparoskopi için operasyon süreleri benzerdir (26). Robot yardımlı laparoskopi için ek laparoskopi deneyimi gerekmez ve öğrenme eğrisi Sorensen ve ark.'larına göre 15-20 efektif ve güvenli vaka sonucudur (27). Braga ve ark.'ları yayımladıkları metaanalizde robot yardımlı laparoskopik yöntem ile standart laparoskopik tekniği karşılaştırdıklarında, hastanede kalış ve operasyon sürelerini daha kisa olarak tespit etmelerine karşın; operasyon başarısı ve komplikasyon oranları açısından fark saptamamışlardır (28). Artan deneyim ile daha komplike girişimler yapılmaktadır. Örneğin Passerotti ve ark.'larının 6 hastalık başarısız açık cerrahi sonrası robot yardımlı laparaskopi uyguladıkları hastalarda herhangi bir komplikasyon tespit edilmemiş ve tüm hastalar postoperatif 2 . gün taburcu edilmişlerdir (29). Benzer şekilde, Hemal ve ark.'ları da başarısız açık cerrahi sonrası ortalama yaşları 13.2 yıl olan 6 hastada komplikasyonsuz olarak robotik pyeloplasti yapmışlar ve açık cerrahi gereksinimi duymadıklarını bildirmişlerdir (30).

\section{Üreterokalikostomi}

Üreterokalikostomi başarısız pyeloplasti girişimi sonrası kalan minimal pelvise tekrar pyeloplasti yapılması yerine kullanılabilecek geçerli bir alternatiftir. Casale ve ark.'ları ortalama yaşı 6.3 yıl olan üreterokalikostomi serilerini yayımlamışlardır (31). Bu seride hiçbir girişimde açığa dönüş ya da komplikasyon saptanmamış ve ortalama operasyon süresi 160 dakika olarak rapor edilmiştir. İki çocuğa eş zamanlı pyelolitotomi yapılmış ve bunun operasyon süresine etkisinin ek olarak 15 dakika olduğu bildirilmiştir. Tüm çocuklara 6-12 ay sonra diüretikli renal sintigrafi yapılmış ve post-operatif obstrüksiyonun bulunmadığı dokümante edilmiştir.

\section{Üreteral reimplantasyon}

Distal üreterde striktür, fibrozis veya radyasyona bağlı değişiklikler ve iatrojenik nedenlere sekonder obstrüksiyon gelişiminde üreteroneosistostomi yapılır. Amaç obstrükte segmentin by pass edilerek üreterin mesaneye reimplantasyonudur. Ayrıca, bilateral yüksek dereceli veya tekrarlayan pyelonefrite yol açan vezikoüreteral reflü (VUR) de üreteroneosistostomi için endikasyondur (32). Ekstravezikal (Lich Gregoir) ve intravezikal (Cohen) teknikler bugün pediatrik populasyonda VUR'nun cerrahi tedavisinde kullanılmaktadır. Ekstra- ya da intravezikal açık cerrahi \%92-98 başarı sonuçlarıyla altın standarttır ve komplikasyon oranları oldukça düşüktür. Ancak anlamlı derecede postoperatif mesane spazmı ve analjezik kullanım gereksinimi vardır $(33,34)$. Son yıllarda yüksek dereceli VUR'nun tedavisinde robot yardımlı üreteral reimplantasyon tekniklerinin kullanımı rapor edilmiştir $(35,36)$.

VUR tedavisinde üreteral reimplantasyon altın standart olmasına rağmen açık cerrahi ile ilişkili postoperatif ağrı, hematüri, mesane irritasyon semptomları, üreter obstrüksiyonu ve persistan veya denovo VUR gibi anlamlı morbiditeler bulunmaktadır (37). Bu morbiditeleri minimalize edebilmek için daha az invaziv girişimlere ihtiyaç doğmuştur. Konvansiyonel laparoskopik üreteral reimplantasyon zor bir yöntemdir ve deneyimli cerrahlar için bile öğrenme eğrisi uzundur. Laparoskopi açık yönteme göre diseksiyon ve sutur atma güçlügü nedeniyle genel kabul görme açısından geride kalmıştır (38). Robot yardımı ile laparoskopi görüş kalitesinin ve aletlerin manevra kabiliyetinin artması ile daha kolay uygulanabilir bir yöntem olmuştur $(17,41)$. Robot yardımlı laparoskopi de açı yöntem gibi; hem ekstra- hem de intravezikal uygulanabilir. Intravezikal yaklaşım bilateral VUR'da veya beraberinde eşlik eden üreterosel, divertikül gibi anomali varsa endikedir. Günümüzde intravezikal yaklaşımla ilgili küçük seriler buunmaktadır. Peter ve Woo 2005 'de rapor ettikleri 6 hastalık serilerinde bilateral intravezikal üreteroneosistostomi başarısını \%91.7 olarak rapor etmişlerdir (35). Yazarlar komplikasyon olarak 1 hastada idrar kaçağı görüldüğünü belirtmişlerdir. Diğer taraftan, 2011'de Marchini ve ark.'ları 19 hastalık robotik intravezikal deneyimlerini, 22 açı intravezikal cerrahi ile grubuyla karşılaştırmışlardır (39). Yazarlar robotik grupta daha az mesane spazmı, hematüri, hastanede kalış ve postoperatif sonda gerekliliği saptamışlardır. Ancak robotik yöntemde daha uzun operasyon süresi gerekmiştir. Robotik grupta 1 hastada üriner retansiyon ve 4 hastada ise; idrar kaçağ 1 görülmüştür. $\mathrm{Bu}$ hastalara ek cerrahi girişim gerekmemiştir. Yazarlar 20 robotik ekstravezikal girişimi 17 açık ekstravezikal girişimle karşılaştırdıklarında mesane spazmı, ağrı, katater kalış ve taburculuk sürelerini benzer olarak bulmuşlardır. Buna ek olarak robotik grupta 2 hastada geçici idrar retansiyonu ve 2 hastada da üreteral idrar kaçağ 1 nedeniyle double J stent gereksinimi duymuşlardır. Sonuçlar teknik olarak robotik ekstravezikal yaklaşımın uygulanabilir olduğunu göstermesine karşın; açık yaklaşımla karşılaştırıldığında morbiditede anlamlı azalma sağlamadığı anlaşılmaktadır.

Intravezikal yöntem ekstravezikal ile kıyaslandığında teknik olarak daha zordur. Pnömovezikum sağlanması trokar kenarlarından veya üreterlerin diseksiyonu sırasında retropreritona kaçak meydana gelmesi sonucu veya küçük çocuklarda yetersiz mesane hacmi $(<130$ cc) nedeniyle güçtür (40). Intravezikal yönteme göre ekstravezikal robot yardımlı üreteral reimplantasyon daha sık kullanılır.

Diğer taraftan, bilateral açık ekstravezikal üreteral reimplantasyon \%10'lara varan geçici üriner retansiyon ile ilişki- 
lidir ve bu nedenle rutin olarak uygulanmamaktadır (24). Robot yardımlı yöntemde 3 boyutlu görüntü ile işeme disfonksiyonu riski daha dikkatli diseksiyon ile minimalize edilebilir (41). Robotik ekstravezikal yaklaşımın başarı oranı açık yaklaşıma benzerdir ancak komplikasyonsuz değildir. On dokuz hastalık bir diğer ekstravezikal robotik üreteral reimplantasyon serisinde, 2 hastada mesaneden idrar kaçağ 1 görülmüş ve 1 hastada üreter obstrüksiyonu saptanmıştır (41).

Robot yardımlı laparoskopik üreteral reimplantasyon şu an başlangıç dönemindedir ve artan deneyim ile komplikasyon oranları ve operasyonun morbiditesi zamanla azalacaktir.

\section{Üreterolitotomi ve Üreteroüreterostomi}

Üreter taşı tedavisinde ekstrakorporeal şok dalga litotripsi (ESWL), perkütan ve endoskopik yöntemler kullanılmaktadır. Ancak bu yöntemler büyük ve impakte taşların tedavisinde yeterli olmayabilir (42). Endoskopik tedavi ile bu taşların temizlenmesi çoklu seanslar gerektirebilir (43). $\mathrm{Bu}$ tedavi modaliteleri ile impakte taşlarda komplikasyon oranları yüksektir (44). Büyük impakte taşlarda üreteroskopik tedavide striktür potansiyel bir komplikasyondur. AUA panelinde büyük impakte taşlarda minimal invaziv tedavilerde başarısızlık durumunda açık üreterolitotomi uygulanabilir bir seçenek olarak kabul edilmiştir (45-48). Açık cerrahide morbidite yüksek olmasına karşın; açık cerrahinin laparoskopik ve robotik eşdeğerleri için kılavuzlarda yeterli veri henüz yoktur. Ancak yine de; minimal invazif üreterolitotominin morbiditesi açık cerrahiye göre anlamlı olarak düşük olduğundan, büyük ve impakte taşların tedavisinde primer tedavi alternatifi olarak kullanilabilir $(47,48)$.

Üreteroüreterostomi için genel olarak transperitoneal yaklaşım tercih edilir ve birçok cerrah retrograd pyelografi sonrası üreter katateri yerleştirdikten sonra işleme devam eder $(49,50)$. Passerotive ve ark.'ları 3 çocukta farklı nedenlere bağlı midüreter darlıklarında üreteroüreterostomi sonuçlarını rapor etmişlerdir (49). Sonuçta her üç vakada da; komplikasyon görülmemiş ve hastalar ortalama 3.5 günde taburcu edilmişlerdir. Retrokaval üreter nedeniyle üreteroüreterostomi serisi de rapor edilmiştir $(51,52)$.

\section{Augmentasyon Sistoplasti ve Mitrofanof Tekniği}

Laparoskopinin alt üriner sistem rekonstrüksiyonunda kullanımı, öğrenme eğrisinin uzunluğu ve potansiyel komplikasyonları nedeniyle uzun zaman almıştır. Ancak son dekatta popülarize olan robot yardımlı cerrahi bu operasyonların oldukça uzun olan öğrenme eğrilerini kısaltmıştır. Buna rağmen literatürdeki deneyim henüz sınırlıdır bazı deneysel çalışmalarla birlikte az sayıda seri ve olgu raporları bulunmaktadır $(53,54)$.

Mitrofanof tekniğinde, hasta litotomi pozisyonunda $25^{\circ}$ Trendelenburg konumundadır. Midklavikular hatta 2 adet çalışma portu, kamera portu ile aynı ya da daha yüksek seviyeye yerleştirilir. Operasyonun başlangıcında tanısal peritonoskopi ile appendiksin uzunluğu ve mobilitesi değerlendirilir. Darlıktan kaçınmak için apendiksin kanlanması mümkün olduğunca korunmalıdır. Bazı yayınlarda çekumdan küçük cuff alınmasının cilt seviyesinde darlık oluşumu- nu azaltabileceği belirtilmiştir (55). Ayrıca, stoma ile submukozal tünelin doğrultusu uyumlu (umblikal stomalarda orta hat detrusotomi, batın sağ alt kadranda ise oblik tünel ile) olmalıdır. Eş zamanlı augmentasyon yapılacak ise apendiksovezikostomi tamamlandiktan sonra gündeme gelir ve stoma mesane posterioruna yapılır (7). Prune-Belly sendromunda ise tam tersine stomanin mesane anteriorunda olmasi teknik olarak daha basittir. Augmentasyon sistoplasti 4. kol ve onun karşısındaki iliak fossada yer alan $12 \mathrm{~mm}$ lik asistan portu dişında pozisyon ve insizyon olarak apendiksovezikostomi ile aynıdır. Augmentasyon sistoplasti için ileoçekal valvin $15 \mathrm{~cm}$ proksimalinden $20 \mathrm{~cm}$ 'lik ileum segmenti alınarak antimezenterik kısmından insize edilerek detübülerize edilir. Ayrılan ileum segmenti sonrası barsak anostomozu 3/0 emilebilir sutur ile uç uca yapılır (56). Augmentasyon için mesane koronal planda açılır ve mesane-barsak anastomozu posteriordan anteriora doğru yapılır. Perkütan suprapubik katater, dren ve mesane boynu kapatılmayacak ise uretral sonda konulurak işlem tamamlanır.

Wille ve ark.'ları 2010'da 3 Prune-Belly hastasındaki appendiksovezikostomi (Mitrofanoff tekniği) deneyimlerini rapor etmişlerdir (57). Bu seride intraoperatif komplikasyon ya da açı̆̆a dönüş belirtilmemiştir. Post-operatif dönemde bir hastada yara yeri enfeksiyonu görülmüştür. Nguyen ve ark.'ları 10 hastalık nörojenik veya miyojenik mesane nedeni ile yapilan appendikovezikostomi serisinde 1 hastada appendiksin yetersiz olması nedeniyle açı̆̆a geçiş, bir hasta da; postoperatif idrar kaçağı nedeniyle açık revizyon gerektiğini bildirmişlerdir (58). Hastaların ikisinde minör idrar kaçırma durumu gelişmiş; bunlardan birisi dekstronamer/hyalunarik asit enjeksiyonuyla; diğeri ise kendiliğinden düzelmiştir. Diğer taraftan, Gundeti ve ark.'ları spina bifida nedeniyle nörojen mesanesi bulunan 6 hastada robot yardımlı enterosistoplasti serisini yayımlamışlardır (59). Bu hasta grubunda, 1 hastada açık cerrahiye geçilmiş, 3 hastada postoperatif komplikasyon (yara yeri enfeksiyonu, alt ekstremite venöz tromboz ve unilateral alt ekstremitede geçici parestezi) gelişmiştir. Sonrasında 3 hastanın stomalarında cilt seviyesinde darlık nedeniyle revizyon gerekmiş; ancak tüm hastalar kontinan olarak bildirilmiştir.

Sonuçta, şu an için robot yardımlı enterosistoplasti deneyimi sınırlıdır. Açık cerrahiye oranla başarı oranı ve avantajlarını (iyileşme süresi, postoperatif ağrı ve estetik görünüm) kıyaslayabilmek için uzun dönem takipleri olan çalışmalara ihtiyaç vardır.

\section{Orşiopeksi}

Robotik cerrahi yüksek intraabdominal yerleşimli ya da iki aşamalı orşiopeksi vakalarında kullanılabilir. İlk defa 2007'de Najmaldin ve Antona bilateral orşiopeksi ve Millerian kanal kalıntılarını eksize ettikleri vaka sunumlarını yayımlamışlardır (60). Operasyon sırasında hasta supin trendelenburg pozisyonundadir ve ayn taraf eleve edilir. Mesanenin boşaltılması ve rektal tüp ile dekompresyon faydalı bulunmuştur (61). Port yerleşimi konvansiyonel laparoskopideki gibidir. Umbilikusa kamera portu, orşiopeksi yapılacak testisle aynı tarafa ve umblikus hizasının biraz üzerinde midklavikular hatta 1 adet ve kontralateralde umblikus altına yine midklavikular hatta 1 adet çalışma portu yerleştirilir. Sutur gereksinimi olmadığından robotik tekniğin laparoskopiye ek üstünlüğ̈̈ yoktur (62). 


\section{Nefrektomi}

Açık nefrektomi ile karşılaştırıldığında laparoskopik teknik hastanede kalış ve analjezik kullanım süresi açısından avantajlıdır. Birçok yazar pediatrik yaş grubunda robotik parsiyel ve total nefrektominin uygulanışını rapor etmişlerdir (61,63-68). Ancak robotik teknik ile açık ve laparoskopik yöntemlerin karşılaştırıldığı bir seri henüz yoktur. Total nefrektomi gibi ekstirpatif girişimlerde maliyet ve hasta tarafından beklenen fayda açısından robot kullanımı sorgulanabilir. Robot kullanımı teknik olarak zor olan parsiyel nefrektomide ise makul bir seçenek olabilir. Robotik sistemdeki etkin görsellik ve artan cerrahi beceri vasküler yaralanma riskini azaltabilir (68).

\section{Sonuç}

Pediatrik yaş grubuda robot kullanımı minimal invaziv cerrahi için önemli bir gelişmedir. Konvansiyonel laparoskopiden farklı olarak üç boyutlu ve daha yüksek çözünürlüklü görüntü, tremorun olmaması, ergonomik pozisyon ve dar anatomik alanda hareket özgürlüğü robot kullanımının avantajları olarak sayılabilir. Öğrenme eğrisinin sonuna, laparoskopi deneyimi olmadan, aynı girişim için ortalama 10 -20 vaka ile erişilebilmektedir $(62,69)$. Ancak maliyeti robotik cerrahinin kullanımında hesaba katılması gereken önemli bir etkendir.

Pediatrik grupta robotik cerrahinin başarı oranları pek çok teknik için konvansiyonel laparoskopi ile benzer olmasına karşın; literatürde robotik ve laparoskopik teknikleri karşılaştıran randomize kontrollü çalışmalar yoktur. Bu çalışmaların yapılması robotik cerrahinin pediatrik grupta uygulama alanlarının netleşmesine katkı sağlayacaktır.

\section{Kaynaklar}

1. Barbosa JA, Barayan G, Gridley CM, Sanchez DC, Passeroti CC, Houck $C$ et al. Parent and patient perceptions of robot vs open surgery scars in children. J Urol. $2013 \mathrm{Jul}$; 190(1):244-50.

2. Meehan JJ, Sandler A. Paediatric robotic surgery: a singleinstitutional review of the first 100 consecutive cases. Surg Endosc. 2008; 22:177-182.

3. Le Bret E, Papadatos S, Folliguet T, et al. Interruption of patent ductus arteriosus in children; robotically assisted versus videothoracoscopic surgery. J Thorac Cardiovasc Surg. 2002;123:973-976.

4. Rahbar R, Ferrari LR, Borer JG, Peters CA. Robotic surgery in the paediatric airway: application and safety. Arch Otolaryngol Head Neck Surg. 2007;133:45-50.

5. Dasgupta P, Fitzpatrick J, Kir R, Gill IS “New Technologies in Urology" Springer, London; 2010.

6. Peters CA. Pediatric robot-assisted pyeloplasty .J Endourol 2011; 25:179-85.

7. Orvieto MA, Large M, Gundeti MS. Robotic paediatric urology. BJU international 2012;110(1):2-13. 2011.

8. Kutikov A, Nguyen M, Guzzo T, Canter D, Casale P. Robot assisted pyeloplasty in the infant lessons learned. J Urol 2006;176:2237-40.

9. Patel NS, Muneer A, Mushtaq I. Laparoscopy as a foundation and its limitations and pitfalls in reconstructive pediatric urology. Pediatric robotic and reconstructive urology: a comprehensive guide. $1^{\text {st }}$ ed. In: Gundeti MS, editor. Chichester, UK: Blackwell Publishing Ltd.; 2012. pp. 51-57.

10. Meininger D, Byhahn C, Markus BH. Total endoscopic Nissen fundoplication with the robotic device "da Vinci" in children. Hemodynamics, gas exchange, and anesthetic management. Anaesthesist 2001;50:271-5.

11. Peters CA. Robotically assisted surgery in pediatric urology. Urol Clin North Am 2004;31:743-52.

12. Cundy TP1, Shetty K, Clark J, Chang TP, Sriskandarajah $\mathrm{K}$, Gattas NE et al. The first decade of robotic surgery in children J Pediatr Surg. 2013 Apr;48(4):858-65.

13. Kasturi S, Sehgal SS, Christman MS. Prospective longterm analysis of nerve- sparing extravesical roboticassisted laparoscopic ureteral reimplantation. Urology 2012;79:680-3.

14. Atug F, Woods M, Thomas R. Robotic assisted laparoscopic pyeloplasty in children. J Urol 2005; 174:1440-1442.

15. Mufarrij PW, Woods M, Shah OD. Robotic dismembered pyleoplasty: a 6-year, multi-institutional experience. J Urol 2008; 180:1391-1396.

16. Schuessler W, Grune M, Tecuanhuey L. Laparoscopic dismembered pyeloplasty. J Urol 1993; 150:1795-1799.

17. Klinger H, Remzi M, Janetschek G. Comparison of open versus laparoscopic pyeloplasty techniques in treatment of uretero-pelvic junction obstruction. Eur Urol 2003; 44:340-345.

18. Gobet R. Pyeloplasty: a transperitoneal approach. Pediatric robotic and reconstructive urology: a comprehensive guide. 1st ed. In: Gundeti MS, editor. Chichester, UK: Blackwell Publishing Ltd.; 2012. pp. 120-124.

19. Wu HY, Canning DA. Robotic surgery outcomes: upper urinary tract Pediatric robotic and reconstructive urology: a comprehensive guide. $1^{\text {st }}$ ed. In: Gundeti MS, editor. Chichester, UK: Blackwell Publishing Ltd; 2012.pp. 290292.

20. Lorenzo F.M. Trevisani, Hiep T. Nguyen. Current controversies in pediatric urologic robotic surgery. Current Opinion in Urology. Jan 2013, 26(7): 871-877.

21. Lee DJ, Kim PH, Koh CJ. Current trends in pediatric minimally invasive urology surgery. Korean J Urol 2010; 51:80-87.

22. Yee DS, Shanberg AM, Duel BP. Initial comparison of robotic-assisted laparoscopic versus open pyeloplasty in children. Urology 2006; 67:599-602.

23. Lee RS, Borer JG. Robotic surgery for ureteropelvic junction obstruction. Curr Opin Urol 2006; 16:291-294.

24. Minnillo BJ, Cruz JA, Sayao RH. Long-term experience and outcomes of robotic assisted laparoscopic pyeloplasty in children and young adults. J Urol 2011; 185:1455-1460.

25. Freilich DA, Penna FJ, Nelson CP. Parental satisfaction after open versus robot assisted laparoscopic pyeloplasty: results from modified Glasgow Children's Benefit Inventory Survey. J Urol 2010; 183:704-708.

26. Franco I, Dyer LL, Zelkovic P. Laparoscopic pyeloplasty in the pediatric patient: hand sewn anastomosis versus robotic assisted anastomosis - is there a difference? J Urol 2007; 178:1483-1486.

27. Sorensen MD, Delostrinos C, Johnson $\mathrm{MH}$, et al. Comparison of the learning curve and outcomes of robotic assisted pediatric pyeloplasty. J Urol 2011;185:2517-2522.

28. Braga LH, Pace K, DeMaria J, Lorenzo AJ. Systematic 
review and meta-analysis of robotic-assisted versus conventional laparoscopic pyeloplasty for patients with ureteropelvic junction obstruction: effect on operative time, length of hospital stay, postoperative complications, and success rate. Eur Urol 2009; 56: 848-57.

29. Passerotti CC, Nguyen HT, Eisner BH, Lee RS, Peters CA. Laparoscopic reoperative pediatric pyeloplasty with robotic assistance. J Endourol 2007; 21:1137-40.

30. Hemal AK, Mishra S, Mukharjee S, Suryavanshi M. Robot assisted laparoscopic pyeloplasty in patients of ureteropelvic junction obstruction with previously failed open surgical repair. Int J Urol 2008; 15: 744-6.

31. Casale P, Mucksavage P, Resnick M, Kim SS. Robotic ureterocalicostomy in the pediatric population. J Urol 2008; 180: 2643-8.

32. Wheeler D, Vimalachandra D, Hodson E. Interventions for primary vesicoureteral reflux. Cochrane Database Syts Rev 2004:CD001532.

33. Cohen SJ. The Cohen reimplantation technique .Birth Defects Orig Artic Ser 1977; 13: 391.

34. Zubieta R, L ó pez PJ. Surgical technique for extravesical vesicoureteral neoimplantation. Arch Esp Urol 2008; 61: 873-81.

35. Peters C, Woo R. Intravesical robotically assisted bilateral ureteral reimplantation. J Endourol 2005; 19:618-621.

36. Olsen L, Deding D, Yeung C. Computer-assisted laparoscopic pneumovesical ureteral reimplantation: Cohen initial experience in pig model. APMIS 2003; (Suppl 109):23-25.

37. Misseri R, Kaefer M. Robotic surgery outcomes: lower urinary tract. Pediatric robotic and reconstructive urology: a comprehensive guide. $1^{\text {st }}$ ed. In: Mohan SG, editor. Chichester, UK: Blackwell Publishing Ltd; 2012. pp. 293-297.

38. DaJusta D, Baker LA. Robotic surgery complications and safety. Pediatric robotic and reconstructive urology: a comprehensive guide. 1st ed. In: Mohan SG, editor. Chichester, UK: Blackwell Publishing Ltd; 2012. pp. 279289.

39. Marchini GS, Hong YK, Minnillo BJ. Robotic assisted laparoscopic ureteral reimplantation in children: case matched comparative study with open surgical approach. J Urol 2011; 185:1870-1875.

40. Casale P. Robotic-assisted extravesical ureteral reimplantation. Pediatric robotic and reconstructive urology: a comprehensive guide. 1st ed. In: Mohan SG, editor. Chichester, UK: Blackwell Publishing Ltd.; 2012. pp. 160-162.

41. Peters CA. Robotically assisted surgery in pediatric urology. Urol Clin North Am 2004; 31:743-752.

42. Segura JW, Preminger GM, Assimos DG. Ureteral stones clinical guidelines panel summary report on the management of ureteral calculi. J Urol 1997; 158:1915.

43. Preminger G, Tiselius H, Assimos D. 2007 Guidelines for the Management of Ureteral Calculi. European Urology 2007; 52:1610-1631.

44. Roberts WW, Cadeddu JA, Micali S. Ureteral stricture formation after removal of impacted calculi. J Urol 1998; 159:723-726.

45. Kane CJ, Bolton DM, Stoller ML. Current indications for open stone surgery in an endourology center. Urology $1995 ; 45: 218$.
46. Bichler KH, Lahme S, Strohmaier WL. Indications for open removal of urinary calculi. Urol Int 1997; 59:102.

47. Rayboy A, Ferzil GS, Ioffreda R, Albert PS. Laparoscopic ureterolithotomy. Urology 1992; 39:223.

48. Skreptis K, Doumas K, Siafakas I, Lykourinas M. Laparoscopic versus open ureterolithotomy. A comparative study. Eur Urol 2001; 40:32.

49. Passerotti CC, Diamond DA, Borer JG, Eisner BH, Barrisford G, Nguyen HT. Robot-assisted laparoscopic ureteroureterostomy: description of technique. J Endourol 2008; 22: 581-5.

50. Smith KM, Shrivastava D, Ravish IR, Nerli RB, Shukla AR. Robot-assisted laparoscopic ureteroureterostomy for proximal ureteral obstructions in children. J Pediatr Urol 2009; 5: 475-9.

51. Yee DS, Shanberg AM. Robotic-assisted laparoscopic ureteroureterostomy in an adolescent with an obstructed upper pole system and crossed renal ectopia with fusion Urology 2006; 68: 673.e5-7.

52. Gundeti MS, Duffy PG, Mushtaq I. Robotic-assisted laparoscopic correction of pediatric retrocaval ureter .J Laparoendosc Adv Surg Tech A 2006; 16:422-4.

53. Passerotti CC, Nguyen HT, Lais A, et al. Robot-assisted laparoscopic ileal bladder augmentation: defi ning techniques and potential pitfalls. J Endourol 2008; 22:35560.

54. Marchetti P, Razmaria A, Zagaja GP, Gundeti MS. Management of the Ventriculo-Peritoneal Shunt in Pediatric Patients During Robot-Assisted Laparoscopic Urologic Procedures. J Endourol 2011; 25: 225-9.

55. Harris CF, Cooper CS, Hutcheson JC, Snyder HM III. Appendicovesicostomy: the Mitrofanoff procedure-a 15-year perspective. J Urol 2000; 163: 1922-5.

56. Gundeti MS, Eng MK, Reynolds WS, Zagaja GP. Pediatric robotic-assisted laparoscopic augmentation ileocystoplasty and Mitrofanoff appendicovesicostomy: complete intracorporeal - initial case report. Urology2008; 72: 1144-7.

57. Wille MA, Jayram G, Gundeti MS. Feasibility and early outcomes of roboticassisted laparoscopic Mitrofanoff appendicovesicostomy in patients with prune belly syndrome. BJU Int 2012; 109:125-129.

58. Nguyen HT, Passerotti CC, Penna FJ. Robotic assisted laparoscopic Mitrofanoff appendicovesicostomy: preliminary experience in a pediatric population. J Urol 2009; 182:1528-1534.

59. Gundeti MS, Acharya SS, Zagaja GP, Shalhav AL. Paediatric robotic-assisted laparoscopic augmentation ileocystoplasty and Mitrofanoff appendicovesicostomy (RALIMA): feasibility of an initial experience with the University of Chicago technique. BJU Int 2011; 107:962969.

60. Najmaldin A, Antao B. Early experience of tele-robotic surgery in children. Int J Med Robot 2007;3:199-202.

61. Passerotti C, Craig A. Peters Robotic-assisted laparoscopy applied to reconstructive surgeries in children. World Journal of Urology. 2006; 24: 193-197.

62. Chaussy Y, Becmeur F, Lardy H, Aubert D. Robot-assisted surgery: current status evaluation in abdominal and urological pediatric surgery.J Laparoendosc Adv Surg Tech A. 2013 Jun;23(6):530-8. doi: 10.1089/lap.2012.0192.

63. Sorensen MD, Johnson MH, Delostrinos C. Initiation 
ofa pediatric robotic surgery program: Institutional challengesand realistic outcomes. Surg Endosc 2010;24:2803-2808.

64. Alqahtani A, Albassam A, Zamakhshary M. Robot assisted pediatric surgery: How far can we go? World J Surg 2010;34:975-978.

65. Volfson IA, Munver R, Esposito M. Robot-assisted urologic surgery: Safety and feasibility in the pediatric population. J Endourol 2007;21:1315-1318.

66. Pedraza R, Palmer L, Moss V. Bilateral robotic assisted laparoscopic heminephroureterectomy. J Urol 2004;171:2394-2395.

67. Olsen LH, Jorgensen TM. Robotically assisted retroperitoneoscopic heminephrectomy in children: Initial clinicalresults. J Pediatr Urol 2005;1:101-104.
68. Lee RS, Sethi AS, Passerotti CC. Robot assisted laparoscopic partial nephrectomy: A viable and safe option inchildren. J Urol 2009;181:823-828; discussion 828-829.

69. O'Brien ST, Shukla AR. Transition from open to roboticassistedpediatric pyeloplasty: A feasibility and outcomestudy. J Pediatr Urol 2012;8:276-281.

Yazışma Adresi:

Bülent Önal

İstanbul Üniversitesi, Cerrahpaşa Tıp Fakültesi,

Üroloji Anabilim Dall, Fatih, İstanbul

Tel: +90 5437955565

e-mail: drbulentonal@gmail.com 